\title{
Simple excision and closure of a distal limb of loop colostomy prolapse by stapler device
}

\author{
K. Masumori $\cdot$ K. Maeda $\cdot$ Y. Koide $\cdot$ T. Hanai $\cdot$ \\ H. Sato $\cdot$ H. Matsuoka $\cdot$ H. Katsuno $\cdot$ T. Noro
}

Received: 16 May 2011/ Accepted: 17 October 2011/Published online: 9 November 2011

(C) The Author(s) 2011. This article is published with open access at Springerlink.com

\begin{abstract}
Stomal prolapse is one of the common complications in transverse colostomy and can be managed conservatively in most cases; however, laparotomy and reconstruction of the stoma may sometimes be required, especially in case of irreducible colostomy prolapse. We have reported a simple local repair with reconstruction of the loop colostomy. We herein report a new more simple technique to avoid laparotomy and allow excision of the irreducible colostomy prolapse and complete closure of the distal limb of loop colostomy when no decompression is required in the distal limb of the stoma. In this procedure, the number of stapler and the time with blood loss for the operation can be saved.
\end{abstract}

Keywords Stoma prolapse - Complication of stoma . Stoma correction $\cdot$ Stoma closure

\section{Introduction}

Prolapse is a common complication in patients with transverse colostomy, and it often occurs in the distal limb [1-5]. The prolapse can be managed conservatively in most cases; however laparotomy and reconstruction of the stoma may sometimes be required, especially in case of irreducible colostomy prolapse. We have reported a simple method to avoid laparotomy and allow a local repair with reconstruction of the loop colostomy [6]. We herein report

K. Masumori · K. Maeda ( $₫)$ - Y. Koide - T. Hanai · H. Sato · H. Matsuoka $\cdot$ H. Katsuno - T. Noro

Department of Surgery, Fujita Health University School of Medicine, 1-98, Kutsukake, Toyoake, Aichi 470-1192, Japan e-mail: kmaeda@fujita-hu.ac.jp a new more simple technique to avoid laparotomy and allow excision of the irreducible colostomy prolapse and complete closure of the distal limb of loop colostomy when no decompression is required in the distal limb of the stoma.

\section{Operative technique}

The operation was performed under venous anesthesia. The prolapsed distal limb of the stoma was grasped by the two Babcock forceps and lifted up (Fig. 1a). Stapler device $\left(\right.$ GIA $^{\text {TM }}$ 80-4.8 stapler; Covidien, Mansfield, MA, USA) was applied to the distal limb of the prolapsed stoma at $1-2 \mathrm{~cm}$ height from the skin level and fired (Fig. 1b). Complete hemostasis was performed with absorbable threads (Fig. 1c). After excision of the distal limb of prolapsed colostomy, the stoma is properly formed (Fig. 1d).

Two patients with diverting loop transverse colostomy underwent this new procedure. One patient who had irreducible colostomy prolapse $10 \mathrm{~cm}$ in length with necrosis in the distal limb (Fig. 1a) had received low anterior resection with covering loop colostomy for rectal cancer 3 months ago and underwent emergent operation with this procedure. The stapler was applied longitudinally to the transverse colostomy in this patient (Fig. 1b). The other patient with vertebral stenosis and hydromyelia had undergone stoma construction for fecal incontinence, severe decubitus and extended sphincter defect 2.5 years ago in another hospital. The patient had repeated stomal prolapse and underwent button-pexy [7] for colostomy prolapse three times in our institute but in vain. Both limbs of the transverse colostomy were prolapsed in this patient, and the irreducible prolapsed stoma in the distal limb was $20 \mathrm{~cm}$ in length when the patient came to the emergency 
Fig. 1 Simple correction of loop colostomy prolapse in the distal limb by stapler device. a The prolapsed distal limb of the stoma was grasped by the two Babcock forceps and lifted up. b Stapler device was applied to the distal limb of the prolapsed stoma at $1-2 \mathrm{~cm}$ height from the skin level and fired. c Complete hemostasis was performed with absorbable threads. d Corrected stoma prolapse
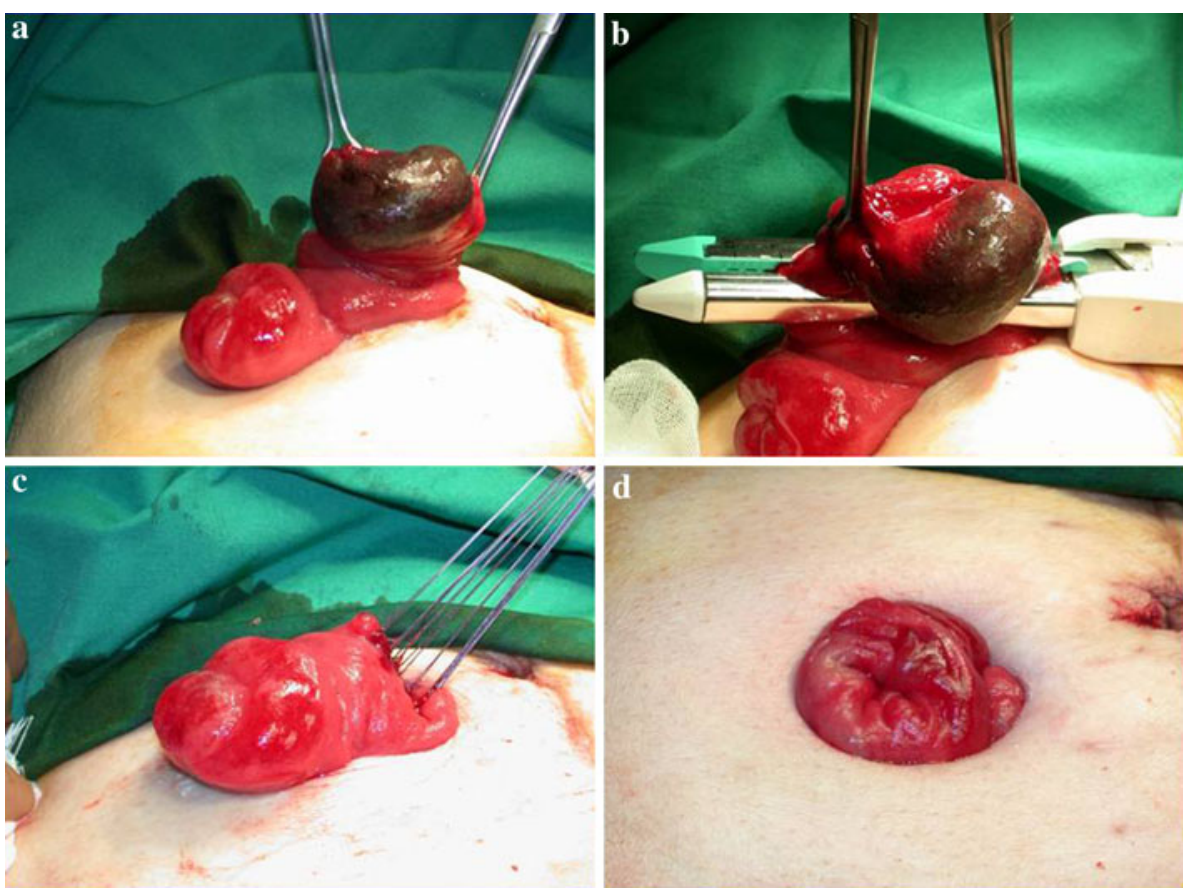

room and was operated. The stapler was applied vertically to the transverse colostomy in this patient. The stapler was used once in each case. Operative times were 25 and $30 \mathrm{~min}$, respectively, and blood loss was negligible in both cases. The postoperative course was uneventful in each case. The first case underwent stoma closure 7 months later without any event. No recurrent prolapse was seen in the second case for 4 years.

\section{Discussion}

Transverse colostomy prolapse occurs when redundant colon invades the stoma with an abdominal pressure [8]. When irreducible condition occurs in the prolapsed stoma, open laparotomy or new stapler techniques $[6,9,10]$ might be required. New stapler techniques are useful for stomal prolapse as a minimally invasive procedure saving operative time and blood loss. Compared to two or three staplers that are usually needed to accomplish these procedures, only one stapler is enough in this new stapler technique, which is cost-effective. Furthermore, operative time in this procedure was almost half of that needed for previous stapler technique with minimal blood loss [6]. Decompression of the distal colon was secured in the previous stapler technique due to the continuity of the distal limb. On the other hand, distal limb is closed in the new technique; therefore, this new technique is not suitable for covering loop stoma with stenosis or obstruction in the distal part of the colon and rectum. As an additional advantage, this technique might be useful for the covering loop stoma to avoid inflow into the distal limb-like second case. In this new procedure, redundant prolapsed colon is excised, which means elimination of the mechanism of stomal prolapse. No recurrence was observed for 4 years in the second case; however, long follow-up time is required to confirm the recurrence. Axis of stapler application to the colon was different in the first and second cases. Vertical application to the transverse colostomy seems more appropriate from our experience because formation of the stoma was more natural after excision. The techniques may be an option for the management of a prolapsed transverse colostomy, especially in case of emergent situation to spare stapler, time and blood loss as a more minimally invasive procedure when no decompression of distal part of the colon and rectum is required.

Conflict of interest The authors declare that they have no conflict of interest.

Open Access This article is distributed under the terms of the Creative Commons Attribution Noncommercial License which permits any noncommercial use, distribution, and reproduction in any medium, provided the original author(s) and source are credited.

\section{References}

1. Fucini C (1989) A simple device for prolapsing loop colostomies. Dis Colon Rectum 32:534-535

2. Docerneck RC (1991) Revision and closure of the colostomy. Surg Clin North Am 71:193-201

3. Londono-Schimmer EE, Leong APK, Phillips RKS (1994) Life table analysis of stomal complications following colostomy. Dis Colon Rectum 37:916-920 
4. Makela JT, Turku PH, Laitinen ST (1997) Analysis of late stomal complications following ostomy surgery. Ann Chir Gynecol 86:305-310

5. Park JJ, Del Pino A, Orsay CP et al (1999) Stoma complications. Dis Colon Rectum 42:1575-1580

6. Maeda K, Maruta M, Utsumi T et al (2004) Local correction of a transverse loop colostomy prolapse by stapler device. Tech Coloproctol 8:45-46

7. Canil BK, Fitzgerald P, Lau G, Cameronn G, Walton M (1995) Button-pexy fixation for repair of ileostomy and colostomy prolapse. Pediatr Surg 30:1148-1149
8. Maeda K, Maruta M, Ustumi T, Sato H, Masumori K, Aoyama H (2003) Pathophysiology and prevention of loop stomal prolapse in the transverse colon. Tech Coloproctol 7:108-111

9. Tepetes K, Spyridakis M, Hatzitheofilou C (2005) Local treatment of a loop colostomy prolapse with a linear stapler. Tech Coloproctol 9:156-158

10. Ferguson HJM, Bhalerao S (2010) Correction of end colostomy prolapse using a curved surgical stapler, performed under sedation. Tech Coloproctol 14:165-167 